\title{
Aerodynamic-Parameter Identification and Attitude Control of Quad-Rotor Model with CIFER and Adaptive LADRC
}

\author{
Sen Yang ${ }^{1,2}$, Leiping $\mathrm{Xi}^{1}$, Jiaxing $\mathrm{HaO}^{3}$ and Wenjie Wang ${ }^{4^{*}}$ (D)
}

\begin{abstract}
Current research on quadrotor modeling mainly focuses on theoretical analysis methods and experimental methods, which have problems such as weak adaptability to the environment, high test costs, and long durations. Additionally, the PID controller, which is currently widely used in quadrotors, requires improvement in anti-interference. Therefore, the aforementioned research has considerable practical significance for the modeling and controller design of quadrotors with strong coupling and nonlinear characteristics. In the present research, an aerodynamic-parameter estimation method and an adaptive attitude control method based on the linear active disturbance rejection controller (LADRC) are designed separately. First, the motion model, dynamics model, and control allocation model of the quad-rotor are established according to the aerodynamic theory and Newton-Euler equations. Next, a more accurate attitude model of the quad-rotor is obtained by using a tool called CIFER to identify the aerodynamic parameters with large uncertainties in the frequency domain. Then, an adaptive attitude decoupling controller based on the LADRC is designed to solve the problem of the poor anti-interference ability of the quad-rotor and adjust the key control parameter $b_{0}$ automatically according to the change in the moment of inertia in real time. Finally, the proposed approach is verified on a semi-physical simulation platform, and it increases the tracking speed and accuracy of the controller, as well as the anti-disturbance performance and robustness of the control system. This paper proposes an effective aerodynamic-parameter identification method using CIFER and an adaptive attitude decoupling controller with a sufficient anti-interference ability.
\end{abstract}

Keywords: Quad-rotor, Qarameters identification, CIFER, Adaptive LADRC

\section{Introduction}

The advantages of quad-rotor unmanned aircraft include their simple structure, easy operation, and small size. They can hover, achieve vertical takeoff, landing, and assume other specific flight postures when the rotating speeds of the four motors are controlled. To complete specific flight missions with high standards, it is necessary to design a control system and establish an accurate model [1-3].

\footnotetext{
${ }^{*}$ Correspondence: wangwenjie@bit.edu.cn

${ }^{4}$ School of Aerospace Engineering, Beijing Institute of Technology, Beijing 100081, China

Full list of author information is available at the end of the article
}

The quad-rotor model is mainly developed using two types of methods. The first is the mechanism analysis method, in which traditional engineering experience and modern aerodynamics knowledge are exploited for analysis and mathematical techniques are used for deriving theoretical models. However, the environmental effects on the model parameters are ignored. It is difficult to ensure the accuracy and complexity of the model simultaneously. The second type of method is the experimental method, e.g., a wind-tunnel experiment or parameter identification experiment. Obtaining the key parameters of a quad-rotor via a wind-tunnel experiment is expensive and time-consuming. Parameter identification can be divided into three methods: "black box," "grey box," 
and "white box." Among them, "grey box" refers to an identification experiment conducted when some mechanisms remain unclear. At present, "grey box" mechanism analysis is an effective method for obtaining the key parameters and establishing accurate models of a quadrotor [4]. In the process of parameter identification, the most commonly used methods are time-domain identification methods, such as the least-squares method, maximum likelihood method, and intelligent identification algorithm based on neural networks. However, the identification results based on the least-squares method are easily affected by measurement noise [5]. The maximum likelihood method has a high complexity and a large amount of calculations [6]. An artificial neural network can easily fall into a local optimal solution with poor real-time performance [7]. Compared with the foregoing time-domain identification methods, the identification of key parameters in the frequency domain is more suitable for a quad-rotor with high dynamic characteristics and a low signal-to-noise ratio of the flight data. Among such methods, CIFER is the aircraft identification software used by the United States' military. It adopts an advanced frequency-domain identification algorithm, which can estimate the transfer function and state-space model of the system for making the frequency response of the identification model match the frequency response of the actual system as closely as possible [8]. The CIFER identification software package is a mature tool with a high identification accuracy, simple operation, and other advantages and is suitable for the parameter identification of a quad-rotor.

A good control system is essential for unmanned aerial vehicles (UAVs) to complete flight missions. At present, the control algorithms applied to quad-rotors mainly include proportional-integral-derivative (PID) control, backstepping, nested saturation control, fuzzy control, and sliding mode control [9-12]. Among these control theories, with the exception of a few classical controllers (such as the PID controller) that cantheories can be applied in practice, most advanced control only be used for simulations, because of their complexity, and there is still a large distance for actual engineering applications [13-15]. Han proposed the active disturbance rejection controller (ADRC), which inherited the essence of PID based on the error and eliminated error. Absorbing the modern control theory, the system disturbance is estimated in real time by an extended state observer. Then, the system is compensated according to the estimated value [16]. This method can effectively improve the system robustness with an independent accurate model. However, the ADRC has many setting parameters that are not suitable for engineering practice [17-19]. The linear active disturbance rejection controller (LADRC), which is based on the bandwidth of the controller for tuning parameters, simplifies the process and is more convenient for engineering applications [20, 21].

The main contributions of the proposed scheme are as follows. First, the motion model, dynamics model, and control allocation model of the quad-rotor are established using the aerodynamic and Newton-Euler equations. Second, the identification tool CIFER is used to identify the aerodynamic parameters with large uncertainties in the frequency domain, and a more accurate attitude m.del of the quad-rotor is obtained. The third contribution is an adaptive attitude decoupling controller with LADRC designed to solve the problem of the poor anti-interference ability of the quad-rotor, so that the control parameter $b_{0}$ can be automatically adjusted to identify the change in the moment of inertia in real time. $b_{0}$ is the most important parameter in the LADRC design process. However, many studies have considered this parameter constant. A large number of simulations and practical applications have revealed that the parameters, as a function of state, should be timevarying, and better control effects can be obtained by seeking appropriate values [22-24]. In this study, the process of adaptive adjustment is added to the LADRC, allowing it to adjust online with changes in the system parameters and improving the robustness and anti-disturbance of the system.

\section{Quad-Rotor Attitude Model}

The mathematical model of the quad-rotor includes three types: the motion model, dynamic model, and control allocation model [25].

(1) Motion model

The quad-rotor has six-degree-of-freedom movement in space, including three linear motions along the coordinate axis and three rotational motions around the center of mass. Only the attitude system model is studied; thus, the linear motion along the three coordinate axes is not considered. We define $\Theta=\left[\begin{array}{lll}\phi & \theta & \psi\end{array}\right]^{\mathrm{T}}$ and $\omega=\left[\begin{array}{lll}p & q & r\end{array}\right]^{\mathrm{T}}$, where $\Theta$ represents the attitude angle of the quad-rotor in the Earth coordinate system, and $\omega$ represents the angular velocity of the quad-rotor in the airframe system.

Their transformation relation can be expressed as follows:

$$
\omega=R_{\omega}^{b} \dot{\Theta} .
$$

(2) Dynamical model

According to Newton's second law, the attitude dynamics equation of the quad-rotor can be expressed as follows: 


$$
\tau_{f}-\tau_{d}+\tau_{g}=J \dot{\omega}+\omega \times J \omega,
$$

where $J=\operatorname{diag}\left(J_{x}, J_{y}, J_{z}\right)$ is the rotation inertia matrix in the body coordinate system. $J_{x}, J_{y}$, and $J_{z}$ represent the rotation inertias of the roll, pitch, and yaw axes in the body, respectively. $\omega=\left[\begin{array}{lll}\omega_{x} & \omega_{y} & \omega_{z}\end{array}\right]^{\mathrm{T}}$ represents the angular velocities of the three axes in the body coordinate system.

$\tau_{f}=\operatorname{diag}\left(\tau_{x}, \tau_{y}, \tau_{z}\right)$ represents the lifting torque of the body. $\tau_{d}=K_{a f} \omega$ represents the air resistance torque. $K_{a f}=\operatorname{diag}\left(K_{a f x}, K_{a f y}, K_{a f z}\right)$ is the air resistance coefficient.

$\tau_{g}$ represents the torque under the gyroscope effect with the expression $\tau_{g}=\sum_{i=1}^{4} \omega \times J_{M} W . \quad J_{M}$ represents the rotational inertia of the rotating rotor, $\Omega_{i}(i=1,2,3,4)$ represents the angular velocity of the four motors, and $W=\left[\begin{array}{lll}0 & 0 & \Omega_{1}-\Omega_{2}+\Omega_{3}-\Omega_{4}\end{array}\right]^{\mathrm{T}}$.

Taking the roll shaft as an example, Eq. (2) is sorted out as follows:

$$
\begin{aligned}
& \tau_{x}-K_{a f_{x}} \dot{\phi}+J_{M}\left(\Omega_{1}-\Omega_{2}+\Omega_{3}-\Omega_{4}\right)=J_{x} \ddot{\phi}+\dot{\phi} \times J_{x} \dot{\phi}, \\
& \tau_{x}-K_{a f x} p+J_{M} \Omega=J_{x} \ddot{\phi}-q r\left(J_{y}-J_{z}\right), \\
& \ddot{\phi}=\frac{\tau_{x}-K_{a f_{x}} p+J_{M} \Omega+\left(J_{y}-J_{z}\right) q r}{J_{x}} .
\end{aligned}
$$

Similarly, the following can be obtained:

$$
\begin{aligned}
& \ddot{\theta}=\frac{\tau_{y}-K_{a f y} q+J_{M} \Omega+\left(J_{z}-J_{x}\right) p r}{J_{y}}, \\
& \ddot{\psi}=\frac{\tau_{z}-K_{a f z} r+\left(J_{x}-J_{y}\right) p q}{J_{z}},
\end{aligned}
$$

where $\Omega=\Omega_{1}-\Omega_{2}+\Omega_{3}-\Omega_{4}$.

In conclusion, the angular motion model of the quadrotor is expressed as follows:

$$
\left[\begin{array}{c}
\ddot{\phi} \\
\ddot{\theta} \\
\ddot{\psi}
\end{array}\right]=\left[\begin{array}{c}
\frac{\left(J_{y}-J_{z}\right) q r+J_{M} \Omega+\tau_{x}-K_{a f x} p}{J_{x}} \\
\frac{\left(J_{z}-J_{x}\right) p r+J_{M} \Omega+\tau_{y}-K_{a f y} q}{J_{y}} \\
\frac{\left(J_{x}-J_{y}\right) p q+\tau_{y}-K_{a f z} r}{J_{z}}
\end{array}\right] .
$$

(3) Control allocation model

The control input of the quad-rotor attitude angle system is set as $\tau=\left[\begin{array}{lll}\tau_{x} & \tau_{y} & \tau_{z}\end{array}\right]^{\mathrm{T}}$.

For the quad-rotor " + ," the lift torque $\tau$ generated by the rotor can be expressed as follows:

$$
\left[\begin{array}{c}
\tau_{x} \\
\tau_{y} \\
\tau_{z}
\end{array}\right]=U \cdot\left[\begin{array}{l}
\Omega_{1}^{2} \\
\Omega_{2}^{2} \\
\Omega_{3}^{2} \\
\Omega_{4}^{2}
\end{array}\right]=\left[\begin{array}{c}
L K_{t}\left(-\Omega_{2}^{2}+\Omega_{4}^{2}\right) \\
L K_{t}\left(\Omega_{1}^{2}-\Omega_{3}^{2}\right) \\
K_{l}\left(\Omega_{1}^{2}-\Omega_{2}^{2}+\Omega_{3}^{2}-\Omega_{4}^{2}\right)
\end{array}\right],
$$

where $L$ represents the length of the quad-rotor arm, $K_{t}$ is the lift coefficient, and $K_{l}$ is the torque coefficient. $U$ is the control allocation matrix. For the quad-rotor "+," $U$ can be expressed as follows:

$$
U=\left[\begin{array}{cccc}
0 & -L K_{t} & 0 & L K_{t} \\
L K_{t} & 0 & -L K_{t} & 0 \\
K_{l} & -K_{l} & K_{l} & -K_{l}
\end{array}\right] .
$$

\section{Parameter Identification Based on CIFER}

As indicated by the control allocation model, the parameters $L, K_{t}, K_{l}, J_{x}, J_{y}$, and $J_{z}$ need to be known for the quadrotor attitude system. $L$ can be accurately determined using a simple measurement method, but the other parameters cannot be determined directly. Therefore, it is necessary to design an identification algorithm to obtain more accurate values. In this paper, the key parameters of the quad-rotor are obtained via the CIFER identification method.

\subsection{Process of CIFER Identification}

The CIFER algorithm is mainly composed of six core modules: frequency-response identification, multi-input processing, combined window processing, single-channel transfer function identification, state-space equation identification, and time-domain verification. Figure 1 shows the connections between the modules [26, 27].

In general, the identification steps of CIFER can be summarized as follows.

(1) The input sweep signal to the quad-rotor platform collects sweep frequency data and process data to make it conform to the actual test requirements. The frequency response of the input and output is calculated mainly by using the Chip-z transformation to calculate the frequency response of each input and output pair.

(2) The frequency response is calculated as a paired single-input single-output (SISO) system, and additional steps are needed to eliminate the effects of multiple related inputs. Therefore, multi-input identification technology should be adopted to regulate the frequency response and partial coherence.

(3) To maximize the accuracy of identification estimation, the foregoing spectral calculation (SISO and multi-input normalization) must be performed using the optimized composite window technology to obtain a single multiple-input multiple-output composite frequency-response 


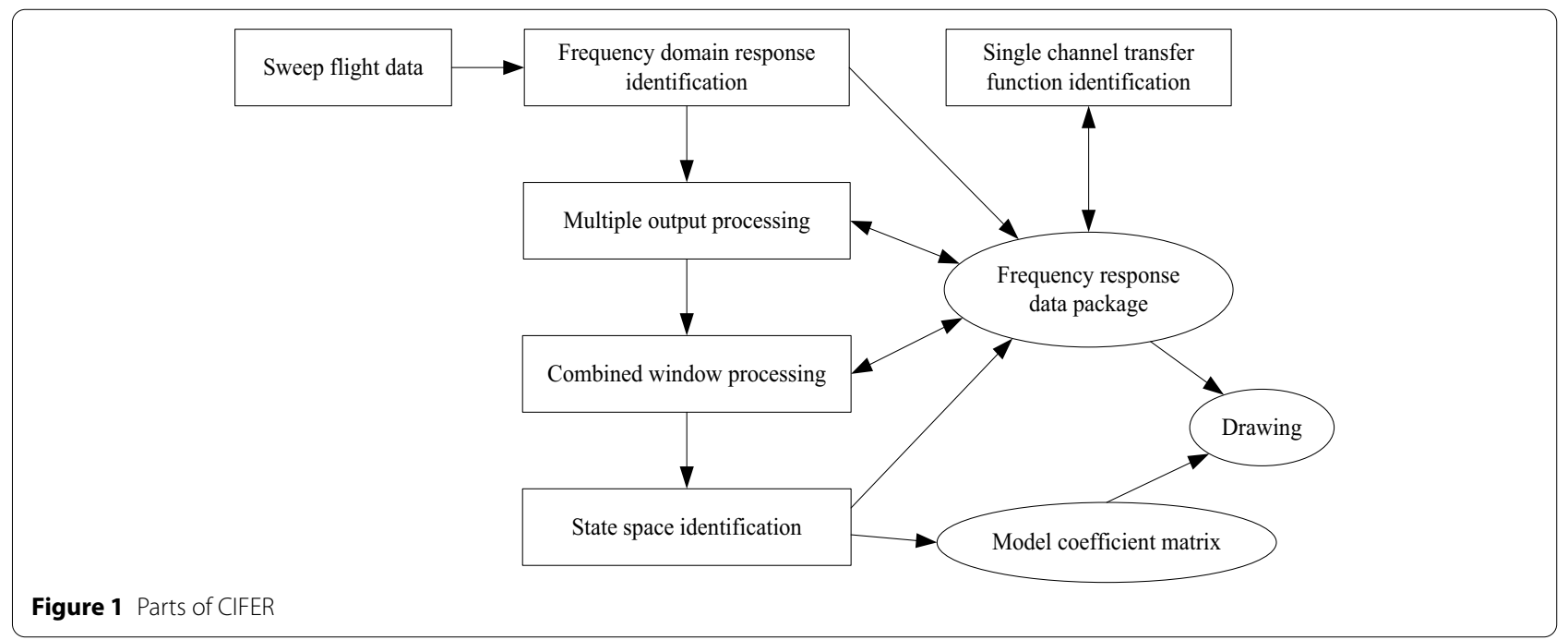

estimation matrix with excellent quality and an excellent dynamic range.

(4) Identify the single-channel transfer function for the processed data and obtain the transfer function of the response.

(5) The identification algorithm is obtained according to the identification criteria, the state-space model of the system is established according to the known parameters, and the initial value of estimation is used for the unknown parameters. The frequency responses of the known and estimated models are matched. Finally, the system state-space equation and the unknown parameter

$$
\hat{T}_{c}=\frac{G_{x y}(f)}{G_{x x}(f)},
$$

where $G_{x y}(f)$ and $G_{x x}(f)$ represent the cross-power spectral function and self-power spectral function, respectively. The objective of system identification is to make the estimation matrix $\hat{T}_{c}$ of the frequency response conform to the actual data matrix. The model adopted through identification is the output error model, and the identification criteria are expressed as follows:

$$
J=\sum_{i=1}^{n_{\omega}} J_{i}=\sum_{i=1}^{n_{\omega}}\left\{\frac{20}{n_{\omega}} \sum_{i=\omega_{1}}^{\omega_{n}} W_{\gamma}\left[W_{g}\left(\left|\hat{T}_{c}\right|-|T|\right)^{2}-W_{p}\left(\angle \hat{T}_{c}-\angle T\right)^{2}\right]\right\},
$$

\subsection{Identification of Key Parameters}

The general form of the linear time-invariant state-space model is expressed as follows:

$$
\begin{aligned}
& \dot{x}=A x+B u(t-\tau), \\
& y=C x+D u(t-\tau) .
\end{aligned}
$$

The Laplace transform of the frequency-response matrix of the system $T(s)$ is expressed as

$$
Y(s)=T(s) U(s),
$$

where $U(s)$ and $Y(s)$ represent the Laplace transforms of the input and output signals, respectively.

The estimation matrix of the frequency-response matrix can be expressed as where $W_{\gamma}=1.58\left(1-e^{-\gamma^{2}}\right) ; n_{\omega}$ represents the number of frequency points; $\omega_{1}$ and $\omega_{n}$ represent the appropriate start and stop frequencies, respectively; $W_{\gamma}$ is a weight function related to the coherent function, with $\gamma^{2}$ being the coherent value at each frequency point; $W_{g}$ and $W_{p}$ are weight functions related to the variance of the amplitude and phase, respectively; and $\hat{T}_{c}$ is the estimation matrix for the frequency response.

\section{Adaptive LADRC Attitude Control Method}

For the quad-rotor system, the rotational inertia is uncertain with changes in the center of gravity, wind disturbance, and other factors, which reduce the tracking accuracy and response speed of the control system. The rotational inertia is identified online, and the 
parameters of the controller are adjusted adaptively according to the obtained moment of inertia; thus, the control system has good anti-interference performance [28-30].

\subsection{Online Identification of Rotational Inertia}

According to the disturbance observer (DOB), the rotational inertia is identified online. By estimating the internal and external disturbances in the model, the real-time rotational inertia is equivalently obtained. Taking the rolling channel as an example, Eq. (5) can be transformed as follows:

$$
J_{x} \ddot{\phi}=\tau_{x}+\tau_{M},
$$

where $\tau_{M}$ represents the unknown disturbance that the DOB needs to estimate, and $\tau_{M}=\tau_{g}-\tau_{f}-\dot{\phi} \times J_{x} \dot{\phi}$. Because the changing rate of the disturbance is far lower than the sampling frequency, $\tau_{M}$ is considered to be constant within a sampling period.

According to Eq. (15), the state-space equation is

$$
\left\{\begin{array}{c}
\dot{x}=A x+B u \\
y=C x
\end{array}\right.
$$

where $x=\left[\begin{array}{ll}\dot{\phi} & \tau_{M}\end{array}\right]^{\mathrm{T}}$ represents the state quantity, $u=\tau_{x}$ represents the control quantity, and $y=\dot{\phi}$ represents the output quantity.

$$
\text { Additionally, } \boldsymbol{A}=\left[\begin{array}{cc}
0 & \frac{1}{J_{x}} \\
0 & 0
\end{array}\right], \boldsymbol{B}=\left[\begin{array}{c}
\frac{1}{\bar{J}_{x}} \\
0
\end{array}\right], \boldsymbol{C}=\left[\begin{array}{ll}
1 & 0
\end{array}\right] \text {. }
$$

According to the state-space equation of Eq. (16), the minimum-order state observer is established to estimate the unknown disturbance:

$$
\left\{\begin{array}{c}
\dot{z}=-\lambda z+\lambda J_{n} \dot{\phi}+u \\
\tau_{M}=-\lambda z+\lambda J_{n} \dot{\phi}
\end{array}\right.
$$

where $z$ is the intermediate variable, $J_{n}$ represents the constant rotational inertia, $M_{c}$ represents the estimated value of the disturbance, and $-\lambda$ represents the observer pole value.

The Laplace transform of Eq. (17) can be expressed as:

$$
M_{c}(s)=J_{n} \lambda \dot{\phi}-\frac{\lambda}{s+\lambda} u(s) .
$$

Two intermediate variables are introduced and expressed as follows:

$$
\left\{\begin{array}{l}
\dot{q}_{0}=-\lambda q_{0}+\lambda \dot{\phi} \\
\dot{q}_{1}=-\lambda q_{1}+\lambda u
\end{array}\right.
$$

By substituting Eq. (19) into Eq. (18), the estimated value of the disturbance can be expressed as $M_{c}=J_{n} \dot{q}_{1}-q_{0}$.

The change in the rotational inertia caused by external factors is denoted as $\Delta J=J_{x}-J_{n}$. Combine it with Eqs. (16)-(18), which can be concluded as follows:

$$
\dot{M}_{c}=-\lambda M_{c}-\lambda\left(\Delta J \phi+K_{a f x} J_{x}+M_{g}\right) .
$$

Substituting the intermediate variables $q_{0}$ and $q_{1}$ into Eq. (20) yields

$$
M_{c}(t)=-\Delta J q_{0}-K_{a f x} q_{0}+M_{g} q_{1} .
$$

Multiplying $q_{0}$ by both sides yields

$$
\dot{q}_{0} M_{c}(t)=-\Delta J q_{0} \dot{q}_{0}-K_{a f x} q_{0} \dot{q}_{0}+M_{g} q_{1} \dot{q}_{0} .
$$

Both $q_{0} \dot{q}_{0} q_{1}$ and $\dot{q}_{0}$ are quadrature, which can be proven. Therefore, Eq. (22) can be used to estimate the change in the rotational inertia:

$$
\Delta J=\frac{\int_{(k-1) T}^{k T} M_{c} \dot{q}_{0} \mathrm{~d} t}{\int_{(k-1) T}^{k T} \dot{q}_{0} \mathrm{~d} t}, \quad k=1,2, \ldots
$$

By adding the rotational inertia of the previous period and the change value of the rotational inertia, the estimated value of the new rotational inertia can be obtained:

$$
\hat{J}_{x}=J_{n}+\Delta J .
$$

A block diagram of the identification process is shown in Figure 2.

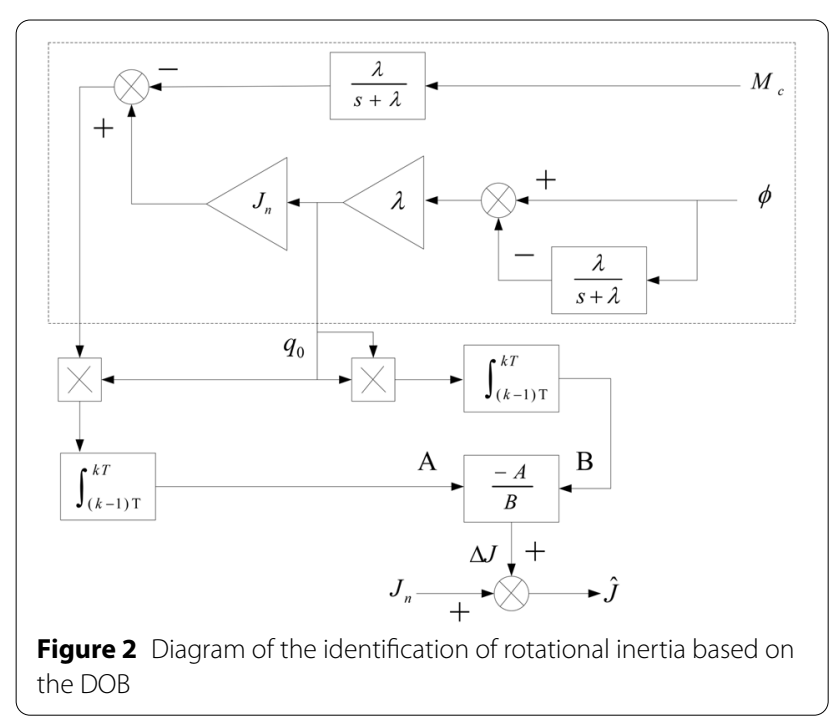




\subsection{Adaptive LARDC Attitude Control Method}

For estimating the rotational inertia online, the control parameters $b_{0}$ can be adjusted according to the rotational-inertia value in real time. The fuzzy logic controller is introduced, and the rules are used to modify the control parameter $b_{0}$ according to the change in the rotational inertia to automatically approach the optimum.

The ratio $\delta$ between the initial value of the rotational inertia and the theoretical value is set as a fuzzy variable in the controller. The variable value of $b_{0}$ is expressed as another fuzzy variable $\Delta b_{0}$. In their domain, eight language subsets are defined: $\left\{\mathrm{P}_{0},{ }^{\text {" }} \mathrm{P}_{1}, \mathrm{P}_{2}, \mathrm{P}_{3}, \mathrm{P}_{3}, \mathrm{P}_{4}, \mathrm{P}_{5}, \mathrm{P}_{5}, \mathrm{P}_{6}\right.$ " " $\mathrm{P}_{7}$ "). The membership function of $\delta$ and $\Delta b_{0}$ is selected as a triangle. According to a large number of simulation experiments, the ratio of the initial value of the rotational inertia to the theoretical value should satisfy $1<\delta<22$, and the change value of the control parameters $b_{0}$ should satisfy $0 \leq \Delta b_{0} \leq 24$. Therefore, the basic theory field of $\delta$ is $(1,22)$, and the basic theory field of $\Delta b_{0}$ is $[0,24]$.

The fuzzy reasoning criterion is described as follows:

If $\delta=P_{i}, \Delta b_{0}=P_{i}(i=0,1,2, \ldots, 7)$. The defuzzification algorithm is the average weight method.

Finally, by adjusting the fuzzy controller, the control parameters $b_{0}^{\prime}$ can be expressed as follows:

$$
b_{0}^{\prime}=b_{0}+\Delta b_{0}
$$

The adjusted control parameters $b_{0}^{\prime}$ can be determined using Eq. (25). By applying the LARDC control rules, the adaptive controller of attitude tracking for a quad-rotor can be obtained, and a corresponding block diagram is shown in Figure 3.

\section{Simulation Results}

\subsection{Experimental Device}

According to the three-degree of freedom (3-DOF) quadrotor platform of the Canadian company Quanser [25], a

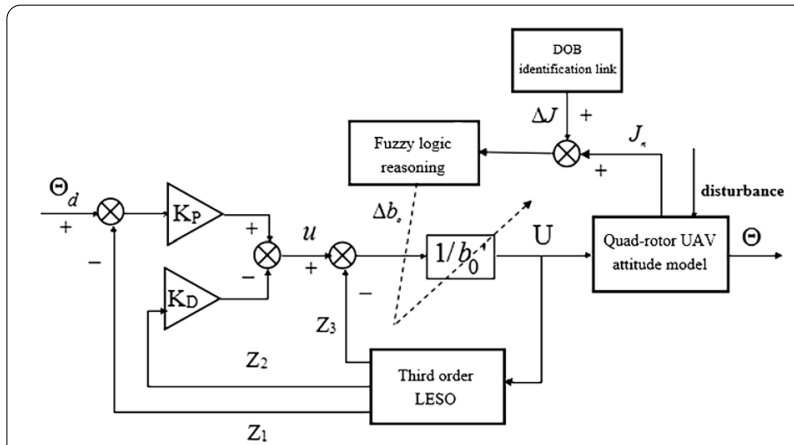

Figure 3 Adaptive controller of attitude tracking for the quad-rotor based on LADRC

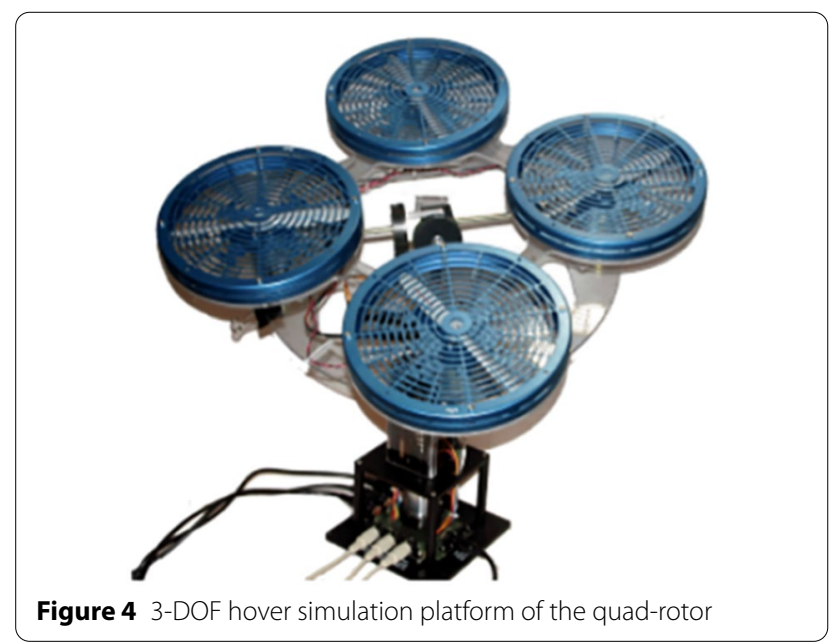

semi-physical simulation environment of the quad-rotor is established, as shown in Figure 4.

The platform consists of four thrusters mounted on a 3-DOF center link, each with a code disc that captures the angle of the aircraft module. The propeller consists of a fan with an impeller, which is driven by a motor. The impeller rotates, generating torque to control the quadrotor for producing roll, pitch, and yaw motions.

The quad-rotor of the 3-DOF platform is distributed in the shape of the symbol "+". The "front" and "back" rotors turn counterclockwise, and the "left" and "right" rotors turn clockwise. The control signal is sent to the quadrotor platform in the form of a voltage by the control program of the quad-rotor on the computer.

\subsection{Parameter Identification}

Owing to the high degree of symmetry of the quad-rotor structure, the differences in the aerodynamic parameters and other aspects of the performance among the motors are very small, and the motors are considered to be approximately equal. The roll channel is taken as an example to illustrate the identification process of CIFER on a 3-DOF semi-physical simulation platform [31, 32].

According to Eqs. (5) and (9), the nonlinear model of the roll channel is expressed as follows:

$$
\ddot{\phi}=\frac{L K_{t}\left(\Omega_{4}^{2}-\Omega_{2}^{2}\right)-K_{a f x} p+J_{M} \Omega+\left(J_{y}-J_{z}\right) q r}{J_{x}} .
$$

Small-angle linearization at the equilibrium point can be approximated as $K_{a f x} \approx 0, J_{M} \approx 0$; when the coupling between channels is ignored, $q r\left(J_{y}-J_{z}\right) \approx 0$.

Then, Eq. (26) can be simplified as follows: 


$$
\ddot{\phi}=\frac{L K_{t}\left(\Omega_{4}^{2}-\Omega_{2}^{2}\right)}{J_{x}},
$$

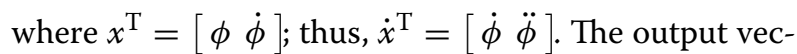
tor is $y=\phi$, and the control vector is $u=\Omega_{4}^{2}-\Omega_{2}^{2}$.

This yields

$$
\left\{\begin{array}{c}
\dot{x}=\left[\begin{array}{ll}
0 & 1 \\
0 & 0
\end{array}\right] x+\left[\begin{array}{c}
0 \\
L \frac{K_{t}}{J_{x}}
\end{array}\right] u, \\
y=\left[\begin{array}{ll}
1 & 0
\end{array}\right] x .
\end{array}\right.
$$

The parameter matrix is given as follows:

$$
A=\left[\begin{array}{ll}
0 & 1 \\
0 & 0
\end{array}\right], B=\left[\begin{array}{c}
0 \\
L \frac{K_{t}}{J_{x}}
\end{array}\right], C=\left[\begin{array}{ll}
1 & 0
\end{array}\right] .
$$

The frequency-sweep signal is input to the experimental platform of the quad-rotor. The frequency bandwidth is predetermined in the frequency-sweep design. Generally, a good bandwidth for quad-rotor identification is at $0.3-12 \mathrm{rad} / \mathrm{s}$. The record length of each scan data should be $4-5$ cycles as $T_{\max }$ of the minimum frequency, and the record length should satisfy $T_{r e c} \geq 4$. After the frequency data are collected, an identification experiment can be performed. The state-space equation of the system is formulated according to the input quantity $u$, state quantity $x$, and output quantity $y$ of the system:

$$
\left\{\begin{array}{c}
M \dot{x}=F x+G u(t-\tau) \\
y=H x+T \bar{x}
\end{array}\right.
$$

where $M, F, G, H, T$, and $\tau$ are the coefficient matrix and coefficients that need to be solved in the CIFER software. In this experiment, the rotor arm length is $L=0.197 \mathrm{~m}$, and the key parameters to be identified are the rotation inertia $J_{x}$ and lift coefficient $K_{t}$. The initial value of the parameter is obtained via prior estimation, and the identification vector $\alpha$ is changed throughout the iterative process until the cost function $J$ is minimized.

After the frequency-domain identification process of the CIFER software package, the model matrices $M, F$, and $G$ are obtained. The identification results for the key parameters are also obtained: $\hat{J}_{x}=0.0492$, $\hat{K}_{t}=0.1170$. A comparison with the values provided in the user manual of the experimental platform $\left(J_{x}=0.0552, K_{t}=0.1188\right)$ indicates that the identification results have small errors (approximately $\pm 10 \%$ for the moment of inertia $\hat{J}_{x}$ and approximately $\pm 1.5 \%$ for the lift coefficient $\hat{K}_{t}$ ).

The experimental results are shown in Figure 5. The frequency response of the data collected by the sweep frequency experiment after the Chip- $\mathrm{Z}$ transformation

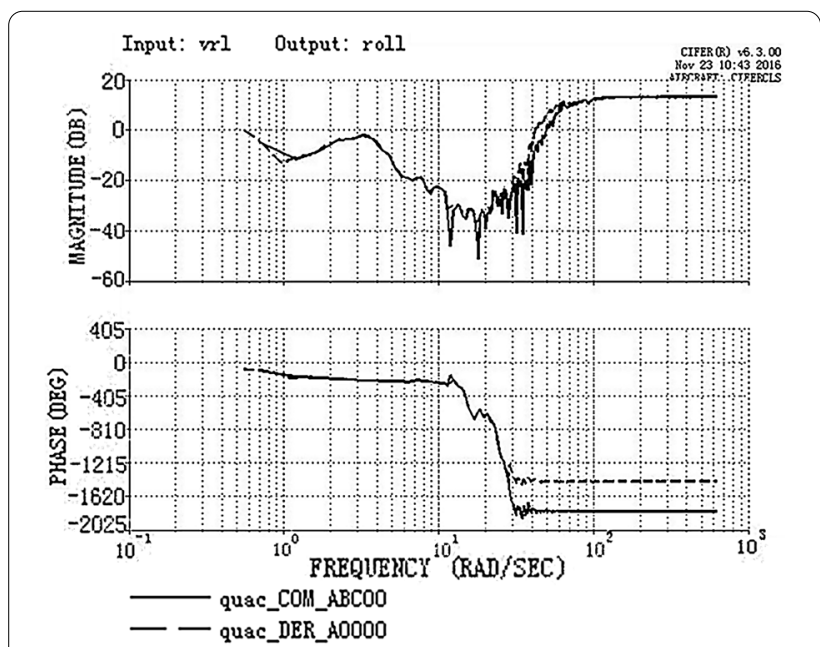

Figure 5 Comparison of the recognition results and experimental data for the frequency response

and combined window processing is automatically generated and saved in a file called "quac_COM_ABCO0" and are plotted as a solid line. The frequency-response data of the identified model were saved in a file called "quac_DER_A0000" and plotted as a dotted line. The upper part of Figure 5 presents an amplitude comparison between the frequency responses of the experimental data and the identification model. The lower half compares the two phases.

The acquisition frequency of the experimental data was $50 \mathrm{~Hz}$. As indicated by the foregoing results, the amplitude and phase of the frequency response were good at the low-frequency stage $(<50 \mathrm{~Hz})$, but jitter occurred at the high-frequency stage $(>50 \mathrm{~Hz})$, and the tracking effect was modest. This may be because additional feedback is needed to maintain the quad-rotor within the operating conditions at high frequencies, and the parameterized model based on mechanism analysis is not adequate to fully describe the system response.

\subsection{Adaptive LADRC Attitude Control}

To verify the performance of the attitude-tracking adaptive controller based on the LADRC, it is compared with PID and the traditional LADRC on a 3-DOF semi-physical simulation platform.

In the MATLAB/Simulink environment, a DOB-based rotational-inertia identification module was developed. The sampling period of the experimental platform was $0.002 \mathrm{~s}$. The test signal was superimposed on the command signal, and the identification module estimated the total disturbance in real time to update the rotationalinertia value. The identification process is shown in Figure 6, where the dashed line indicates the reference value 


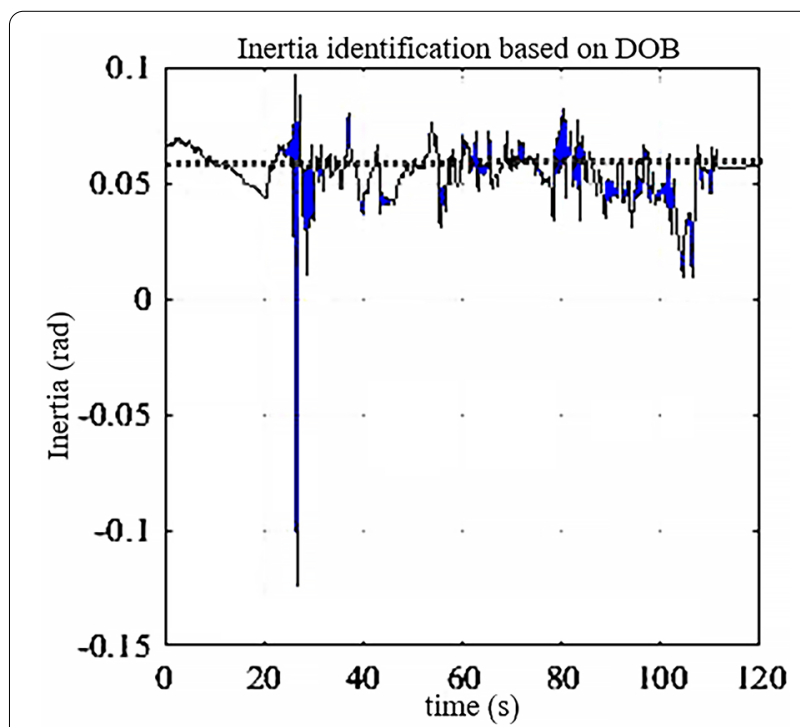

Figure 6 Rotational-inertia identification process

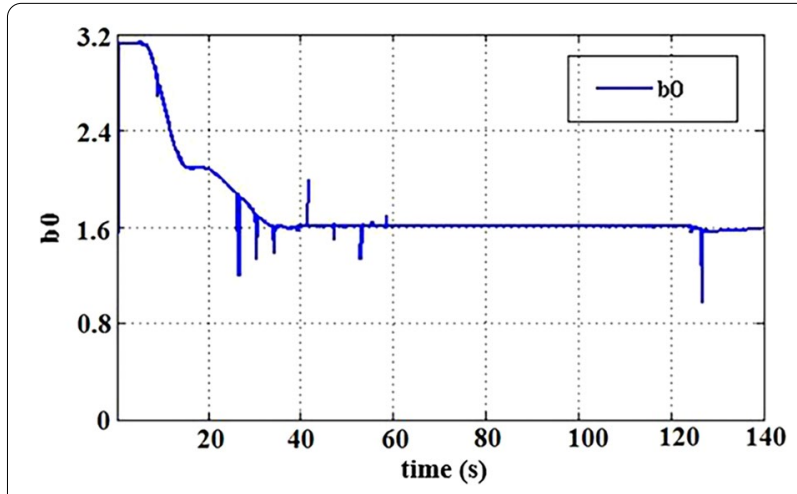

Figure 7 Adjustment process for the control parameter $b_{0}$

of the rotational inertia $\left(0.0552 \mathrm{~kg} \cdot \mathrm{m}^{2}\right)$, and the solid line indicates the identification value. The final identification results indicate that the rotational inertia was stable at $0.0517 \mathrm{~kg} \cdot \mathrm{m}^{2}$, which is higher in the identification accuracy.

In the process of changing with the rotational inertia $J_{x}$, the controller parameters $b_{0}$ were optimized according to the fuzzy control rules. The initial value of $b_{0}$ was set as 3 , and the final value was approximately 1.6. The optimization process is shown in Figure 7, where the curve represents $b_{0}$.

The initial attitude angle of the quad-rotor experimental platform was given as $\left[\begin{array}{lll}\Phi & \theta & \Psi\end{array}\right]=\left[\begin{array}{ll}1^{\circ} \\ \hline\end{array}\right.$ $\left.1^{\circ} 1^{\circ}\right]$, and the desired attitude angle was set as $\left[\begin{array}{lll}\phi_{d} & \theta_{d} & \psi_{d}\end{array}\right]=\left[\begin{array}{lll}4^{\circ} & 4^{\circ} & 4^{\circ}\end{array}\right]$. Taking the roll channel as an example, the bandwidth of the controller was

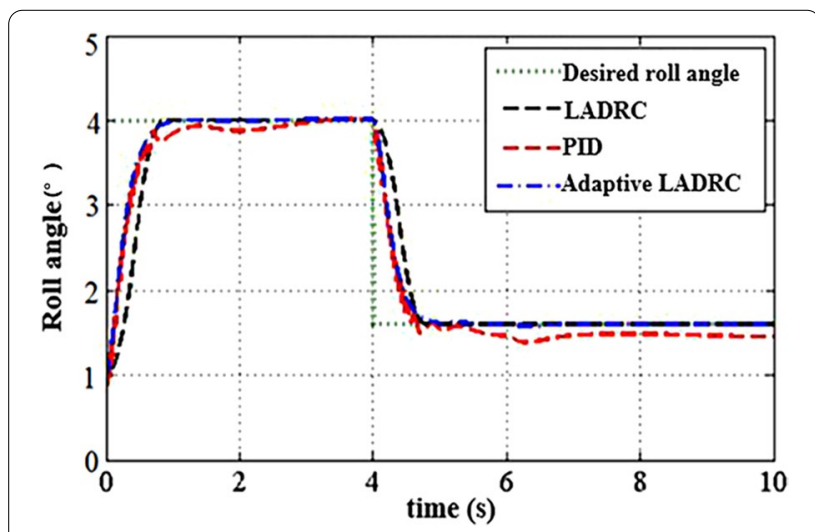

Figure 8 Comparison of three control methods

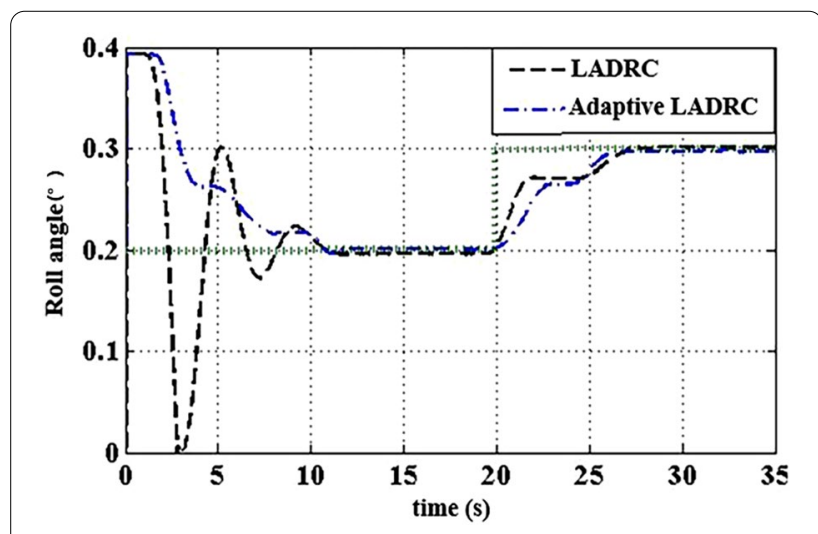

Figure 9 Comparison for small angles

adjusted repeatedly in the LADRC, and $\omega_{c}=10$ was finally selected. The observer bandwidth was $\omega_{0}=30$. In the PID controller, the parameters with good control performance were obtained as $k_{p 1}=0.01, k_{i_{1}}=0.05$, and $k_{d_{1}}=0.15$ after multiple-parameter adjustment. A comparison of the three control methods is shown in Figure 8 , where the dotted line indicates the expected roll angle, the red dashed line indicates the tracking performance by the PID controller, the black dashed line indicates the tracking performance of the original LADRC, and the dashed-dotted line indicates the tracking performance of the adaptive LADRC.

As shown in Figure 8, when the expected roll angle was set within a certain angle range, the adaptive LADRC was not superior to the traditional LADRC with regard to the tracking performance. However, when the angle was small, that is, the desired angle was set as $1^{\circ}$ or $<1^{\circ}$, the adaptive LADRC was clearly superior in tracking. The simulation results are presented in Figure 9, where the dotted line indicates the expected angle, the black dashed line indicates the tracking performance of the LADRC, and the dashed-dotted line 


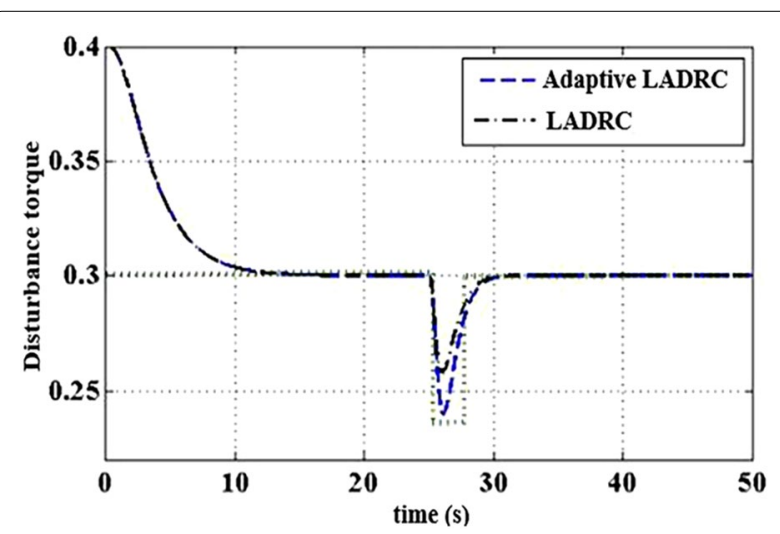

Figure 10 Pulse disturbances of the controllers

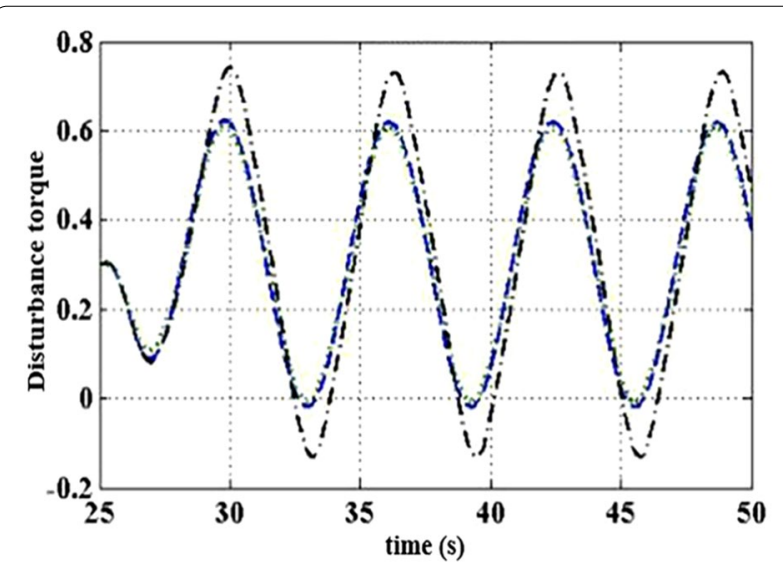

Figure 11 Sinusoidal disturbances of the controllers

indicates the tracking performance of the adaptive LADRC. As shown, the traditional LADRC had a large overshoot and a long response time, whereas the adaptive LADRC had a fast response and small overshoot, indicating that the real-time adjustment of the control parameter $b_{0}$ improved the performance of the LADRC.

To verify the dynamic performance and anti-disturbance of the control system, an impulse disturbance with an amplitude of $0.2 \mathrm{~N} \cdot \mathrm{m}$ and a period of $5 \mathrm{~s}$ and a sinusoidal disturbance with an amplitude of $0.3 \mathrm{~N} \cdot \mathrm{m}$ and a period of $5 \mathrm{~s}$, respectively, were used. The disturbance was estimated by the LADRC and the adaptive LADRC, and the results are shown in Figures 10 and 11. The dotted line indicates the actual disturbance moment, the dashed-dotted line indicates the disturbance estimation results of the LADRC, and the dashed line indicates the disturbance estimation results of the adaptive LADRC.

As shown in Figures 10 and 11, for the impulse disturbance, the estimation provided by the adaptive LADRC was closer to the real value, and the reaction speed was higher. For the sinusoidal disturbance, the estimation provided by the adaptive LADRC had almost no deviation from the actual disturbance, whereas the traditional LADRC had a significant deviation. Compared with the traditional LADRC, the adaptive LADRC can optimize the parameter b0 in real time; thus, the system can estimate the disturbance more accurately, and the performance of the controller is superior.

\section{Conclusions}

A quad-rotor aerodynamic-parameter identification technique based on CIFER and an adaptive LADRC attitude control method was designed. The conclusions and future prospects are summarized as follows.

(1) The motion, dynamics, and control allocation models of the quad-rotor were established according to the aerodynamic theory and Newton-Euler equations, and a CIFER identification environment was developed to identify the key parameters of the quad-rotor model.

(2) To deal with the model uncertainty caused by the change in the moment of inertia, the DOB is used to identify the moment of inertia online, and the control parameters are optimized in real time by the adaptive LADRC depending on the rotation.

(3) Simulation results indicated that the parameter identification method has high precision, that the proposed control design can accurately estimate and compensate for the total disturbance of the system, and that the control process is fast and accurate.

(4) Future research will focus on the multi-channel coupling attitude model and the combination of the LADRC and NLADRC methods.

\section{Acknowledgements}

Not applicable.

\section{Authors' Contributions}

SY and WW were in charge of the whole trial; SY and LX wrote the manuscript; SY and WW revised the manuscript; $L X, J H$, and WW assisted with the sampling and laboratory analyses. All authors read and approved the final manuscript.

\section{Authors' Information}

Sen Yang was born in 1984 and is currently a director and lecturer at Department of UAV Engineering, Army Engineering University, China. His research interests include system parameter identification and adaptive control.

Leiping Xi was born in 1980 and is currently a director and associate professor at Department of UAV Engineering, Army Engineering University, China. His research interests include system parameter identification and sliding mode control (SMC)

Jiaxing Hao was born in 1992 and is currently a PhD candidate at Beijing Institute of Technology, China. He received his Master's degree from Shijiazhuang Tiedao University, China, in 2020.

Wenjie Wang was born in 1988 and is currently an assistant professor at School of Aerospace Engineering, Beijing Institute of Technology, China. His 
research interests include active control systems, fluid dynamics, and acoustic engineering.

\section{Funding}

Supported by National Natural Science Foundation of China (Grant No. 61501493).

\section{Competing Interests}

The authors declare no competing financial interests.

\section{Author Details}

${ }^{1}$ Department of UAV Engineering, Army Engineering University, Shijiazhuang 050003, China. ${ }^{2}$ School of Automation Science and Electrical Engineering, Beihang University, Beijing 100083, China. ${ }^{3}$ School of Electrical and Electronic Engineering, Shijiazhuang Tiedao University, Shijiazhuang 050043, China. ${ }^{4}$ School of Aerospace Engineering, Beijing Institute of Technology, Beijing 100081, China.

Received: 6 December 2019 Revised: 15 November 2020 Accepted: 30 November 2020

Published online: 04 January 2021

\section{References}

[1] Róbert Szabolcsi. The quadrotor-based night watchbird UAV system used in the force protection tasks. International Conference Knowledge-Based Organization, 2015, 21(3): 170-184.

[2] Omid Mofid, Saleh Mobayen. Adaptive sliding mode control for finitetime stability of quad-rotor UAVs with parametric uncertainties. ISA Transactions, 2018.

[3] Lebsework Negash, Sang-Hyeon Kim, Han-Lim Choi. An eigenstructure assignment embedded unknown input observe approach for actuator fault detection in quadrotor dynamics. IFAC Papers OnLine, 2016, 49(17): 426-431.

[4] Yousaeng Lee, Seungjoo Kim, Jinyong Suk, et al. System identification of an unmanned aerial vehicle from automated fight tests. AIAA-2002-2003, 2002.

[5] Hanbing Li, Dawei Wu. An approach of UAV's aerodynamic parameter identification. Flight Dynamics, 2014, 32(2): 183-188.

[6] Congkui Hao. The attitude control system and control method of quad-rotor. North China University of Technology, 2014.

[7] Juping Wang. Research on parameter identification of permanent magnet synchronous motor based on neural network. Shanghai: Donghua University, 2016.

[8] Yu Zou, Hailong Pei, Xin Liu. Study on CIFER algorithm, a method for frequency identification of aircraft model. Electronic Optics \& Control, 2010, 17(5): 46-49.

[9] Jing-Jing Xiong, Guo-Bao Zhang. Global fast dynamic terminal sliding mode control for a quadrotor UAV. ISA Transactions, 2017, 66: 233-240.

[10] S Gilbert, E Varghese. Design and simulation of robust filter for tracking control of quadcopter system. 2017 International Conference on Circuit, Power and Computing Technologies (ICCPCT), 2017.

[11] S K Kim, C K Ahn. Auto-tuner based controller for quadcopter attitude tracking applications. IEEE Transactions on Circuits and Systems II: Express Briefs, 2019, 66(12): 2012-2016.

[12] H Wang, XYe, Y Tian, et al. Attitude control of a quadrotor using model free based sliding model controller. International Conference on Control Systems and Computer Science, IEEE, 2015: 149-154.

[13] W C Lee, H L Choi. Interactive multiple neural adaptive observer based sensor and actuator fault detection and isolation for quadcopter. 2019 International Conference on Unmanned Aircraft Systems (ICUAS), 2019.

[14] Chun Kiat Tan, Jianliang Wang, Yew Chai Paw, et al. Tracking of a moving ground target by a quadrotor using a backstepping approach based on a full state cascaded dynamics. Applied Soft Computing, 2016: 47-62.

[15] H Liu, X Wang, Y Zhong. Quaternion-based robust attitude control for quadrotors. IEEE Transactions on Industrial Informatics, 2015, 11(2): 406-415.
[16] Jingqing Han. Auto disturbances rejection controller and its applications. Control and Decision, 1998, 13(1): 19-23.

[17] X Liang, J Li, F Zhao. Attitude control of quadrotor UAV based on LADRC method. 2019 Chinese Control and Decision Conference (CCDC), 2019.

[18] Jie Li, Xiaohui Qi, Yuanqing Xia, et al. On asymptotic stability for nonlinear ADRC based control system with application to the ball-beam problem. Proceedings of the American Control Conference, 2016.

[19] Wenchao Xue, Yi Huang. Tuning of sampled-data ADRC for nonlinear uncertain systems. Journal of Systems Science \& Complexity, 2016: 1-25.

[20] Zhiqiang Gao. Scaling and bandwidth-parameterization based controller tuning. Proceedings of the American Control Conference, Denver, Colorado, June4-6, 2003: 4989-4996.

[21] S Zhao, Z Gao. Active disturbance rejection control for non-minimum phase systems. Proceedings of the 29th Chinese Contro/ Conference, Beijing, 2010: 6060-6070

[22] Xing Chen. Active disturbance rejection controller tuning and its applications to thermal processes. Beijing: Tsinghua University, 2008.

[23] S Li, Z Liu. Adaptive speed control for permanent-magnet synchronous motor system with various of load inertia. IEEE Transactions on Industrial Electronics, 2009, 56(8): 3050-3059.

[24] A Morfin-Santana, F M Palacios, I Gonzalez-Hernandez, et al. Robust control for octorotor Unmanned Aerial Vehicle in H-Configuration. 2018 15th International Conference on Electrical Engineering, Computing Science and Automatic Control (CCE), 2018.

[25] M H Jafri, H Mansor, T S Gunawan. Development of fuzzy logic controller for quanser bench-top helicopter. Materials Science and Engineering, 2017: 2-8.

[26] Yu Zou. Frequency identification of UAV based on CIFER. Guangzhou: South China University of Technology, 2015.

[27] M S Rafaq, JW Jung. A comprehensive review of state-of-the-art parameter estimation techniques for permanent magnet synchronous motors in wide speed range. IEEE Transactions on Industrial Informatics, 2020, 16(7): 4747-4758.

[28] B Tian, L Liu, H Lu, et al. Multivariable finite time attitude control for quadrotor UAV: Theory and experimentation. IEEE Transactions on Industrial Electronics, 2017: 1-1.

[29] A Federico, Z Emanuele, M Ammar, et al. Knot-tying with flying machines for aerial construction. IEEE RSJ International Conference on Intelligent Robots and Systems, 2015: 5917-5922.

[30] W Mizouri, S Najar, M Aoun, et al. Modeling and control of a quadrotor UAV. Proceedings of 15th International Conference on Sciences and Techniques of Automatic Control \& Computer Engineering, 2014: 343-348.

[31] J Zhou, X Lyu, X Cai, et al. Frequency domain model identification and loop-shaping controller design for quadrotor tail-sitter VTOL UAVs. ICUAS, 2018: 1142-1149.

[32] X Lyu, H Gu, J Zhou, et al. Simulation and flight experiments of a quadrotor tail-sitter vertical take-off and landing unmanned aerial vehicle with wide flight envelope. International Journal of Micro Air Vehicles, 2018, 10(4): 303-317.

\section{Submit your manuscript to a SpringerOpen ${ }^{\oplus}$ journal and benefit from:}

- Convenient online submission

- Rigorous peer review

- Open access: articles freely available online

- High visibility within the field

Retaining the copyright to your article

Submit your next manuscript at springeropen.com 